\title{
The impact of transitioning from a 24-hour to a 16-hour call model amongst a cohort of Canadian anesthesia residents at McMaster University - a survey study
}

\author{
This article was published in the following Dove Press journal: \\ Advances in Medical Education and Practice \\ 7 August 2015 \\ Number of times this article has been viewed
}

\section{David Sussman \\ James E Paul}

Department of Anesthesia, McMaster University, Hamilton, ON, Canada
Correspondence: James E Paul

Department of Anesthesia (HSC-2V90), McMaster University, 1280 Main Street West Hamilton, ON L8S 4KI, Canada

Tel +I $90552 \mid 2100$ ext 21736

Fax + I 905523 I244

Email paulj@mcmaster.ca
Purpose: The primary objective of this study was to assess anesthesia residents' opinions and perceptions on wellness/burnout, fatigue, education, and patient safety after the initiation of a reduced call model (16-hour call).

Methods: A prospective cohort study was conducted at three time points during the 2013-2014 academic year. A web-based questionnaire consisting of 23 questions was electronically distributed to all anesthesia residents from postgraduate years (PGY) 1 to 5 who were part of the active call roster $(n=84)$ at McMaster University in Hamilton, Ontario. Descriptive summaries were calculated, counts and percentages were used for categorical variables, and answers to open text questions were reviewed for themes.

Results: A response rate of $67 \%$ was obtained for this study. A majority of anesthesia residents (65\%) approved of 16-hour call, felt that their overall quality of life as a senior resident (PGY3 or greater) or junior resident (PGY2 and below) had improved (73\% and 55\%, respectively), and reported overall feeling less fatigued. Most respondents indicated that the quality of education remained unchanged (47\%), or had improved (31\%). And most felt better prepared for the royal college exam (52\%). Most felt patient safety had improved or was unchanged (both $48 \%$ ).

Conclusion: The study demonstrates that 16-hour call improved resident wellness, reduced burnout and fostered an environment where residents are less fatigued and more satisfied with their educational experience promoting an environment of patient safety. Overall, the anesthesia residency group demonstrated that not only is 16-hour call preferred but beneficial.

Keywords: resident wellness, reduced call model, duty hours, patient safety, cohort study, fatigue

\section{Introduction}

Anesthesia is a field that allows for a very small margin of error with a short time interval to comprehend and rectify a problem, or worse, a mistake. Anesthesia involves amalgamating real time data correlated to patient individual conditions and comorbid disease, developing an appropriate individualized plan, and executing this plan under controlled conditions to ensure a favorable patient outcome, all of this under time constraints. At the best of times this is difficult; now imagine performing this after being awake for 24 hours or longer. ${ }^{1,2}$

Scientists have warned that the effects of severe fatigue are comparable to those provoked by alcohol. After 24 hours of sustained wakefulness, the impairment in psychomotor function is equivalent to a blood alcohol concentration of $0.1 \%$ which is 
well above the legal limit for driving a car in Canada or the US. ${ }^{2}$ However, whilst consumption of alcohol is forbidden in the transport industry, fatigue in the operating room (OR) appears to be tolerated. ${ }^{3}$

We know that fatigue kills; we see it in almost all regulated industries, eg, aviation, and transport. Human performance is systematically degraded by inadequate sleep and at unfavorable times in the daily cycle of the circadian biological clock. ${ }^{4-7}$ Although the exact magnitude of the impact of that fatigue on medical errors cannot truly be evaluated, fatigue is cited as a contributing factor in $10 \%$ of wrongdrug errors and $2 \%$ of all anesthesia incidents reported to the New Zealand incident monitoring system. ${ }^{4}$ Furthermore, the literature indicates that, when fatigued (after prolonged wakefulness $>24$ hours), anesthesia residents scored significantly worse on the vigilance tests than rested residents, in particular, on interpreting important clinical information. ${ }^{8}$ These data are important because they suggest that well rested practitioners should help reduce medical error and increase patient safety. Why should it take a fatal wake up call for change to occur in the field of anesthesia? ${ }^{9}$

Considerable controversy exists regarding the optimal work hours of physicians and surgeons in training. It has long been suspected that extended work hours negatively affect medical and surgical performance and have deleterious effects on resident health and well-being. ${ }^{9}$ In July 2003, the Accreditation Council for Graduate Medical Education (ACGME) mandated an 80-hour duty limit averaged over a 4-week period for residents. In 2011, they mandated 16-hour call for all first year residents. Shortly thereafter, Quebec, the only Canadian province thus far, also instituted 16-hour call for all residents in house.

However, a meta-analysis that looked at all the data from 1980 demonstrated that there was no overall improvement in patient outcomes as a result of decreased resident duty hours (RDH). There was no improvement in education related to $\mathrm{RDH}$ restriction. In fact, there was a trend demonstrating worsening pass rates on board examinations for some specialties. Overall, resident wellness appeared to be improved after implementation of an 80-hour work week, but there was little improvement or even a negative effect on wellness after 16-hour maximums were implemented. ${ }^{10,11}$

The current ongoing debate in residency training committees is: what are appropriate RDH? How many hours should residents be working and how will this impact not only residents, but also delivery of health care and patient safety? Although not yet mandated by law, many residencytraining programs in Ontario are making the transition from
24 hours to some form of reduced call model (16 hours, or night float system).

Extensive research in this area has recently examined the issues associated with making such a change, but none have looked at the field of anesthesia specifically. At McMaster University, the residency-training program initiated the transition to 16-hour call at the beginning of the 2013 academic year, and we decided to look at how the rollout of 16-hour call affected resident wellness, education, and patient safety.

The authors believe that overall the anesthesia residents will support 16-hour call because it will provide more opportunity to study and prepare for cases; reduce fatigue which will in turn reduce medical error and promote a better educational environment. Thus, it is hypothesized that the anesthesia residents will prefer a reduced call model over the traditional 24-hour model, and scores in resident wellness fatigue, education, and patient safety will all be improved. This survey study was designed to assess the impact of a reduced call hours (from 24 to 16) model on McMaster anesthesia resident wellness, education, fatigue, time management, and their perception of patient safety.

\section{Methods Design}

This study was approved by the Hamilton Integrated Research Ethics Board. The survey was an adaptation of the already validated survey produced by Drolet et al in the New England Journal of Medicine. ${ }^{10}$ The survey consisted of 23 questions covering demographics, perceptions of the changes in education, patient safety, resident wellness, and education, overall perception of restricted duty hours, practicing status, work hours, and quality of life measures. This survey was chosen as it was evidence based, touched on all the important aspects of residency training goals, and has demonstrated its effectiveness in a similar study population.

The questionnaire was a web-based questionnaire, delivered to anesthesia residents at McMaster University via email. The survey was administered at three time points throughout the academic year: July 2013, December 2013 and June 2014. This was to evaluate and trend resident opinions throughout the three major phases of change (initiation, maintenance, and end of implementation), and to try and assess the effects of the intervening policy change. We suspected that the attitudinal associations may not be stable over time, and the goal of administering the survey at different time intervals was to try and account for this change.

We altered the original survey in a few ways. Three questions were added to look specifically at patient safety in anesthesia. 
Some of the wording of the original survey was altered to make it more applicable to anesthesia and Canadian standards. The possible responses for the original Drolet et al survey ranged from five responses from strongly disagree (1) to strongly agree (5). Our scale was changed to offer three responses: worse (1), unchanged (2), and better (3). The decision to limit responses to three categories was to try and focus resident responses more appropriately to gauge overall opinion.

To minimize potential bias, potential participants were not informed of the specific purpose of the study. Informed consent was obtained by study participants prior to them completing the survey. Given the intimate nature of some questions and the potential revelation of undesirable behaviors, all data were kept anonymous.

\section{Setting}

The McMaster University anesthesia residency-training program is a medium-sized training program affiliated with four tertiary care teaching hospitals that cover all surgical specialties, including: high risk obstetrics, pediatrics, trauma, cardiac, vascular, thoracic, and transplant. Prior to 2013, all residents in anesthesia were doing 24-hour call and had done so since the inception of the training program, more than 40 years ago. At the beginning of the academic year, a reduced call model (16 hours) was rolled out at all the academic hospitals.

\section{Study population}

Participants were all anesthesia residents from McMaster University in Hamilton. Eligible participants were those from the postgraduate year (PGY) 1 to PGY5 who were on the active call roster. Residents were defined as physicians with 5 or fewer years of post-medical school experience. Anesthesia fellows and off-service residents were excluded from the study.

At the time of the survey there were 28 potential participants. Each participant was able to participate in all three surveys distributed at the three time points throughout the year. This would leave a potential response rate of 84 . Participants were contacted via email and asked to complete the web-based survey. To maximize response rate, we sent three sequential emails to the participants 1 day, 3 days and 7 days after the initial contact.

\section{Statistical analysis}

Descriptive summaries were calculated according to age, sex, residency year, marital status, and presence of children. Counts and percentages were used for categorical variables, and answers to open text questions were reviewed for themes.

\section{Results}

In total, 56 responses were obtained (Table 1). Across all test intervals residents' opinions were quite consistent on most questions, making the response rate $67 \%$.

\section{Sixteen hour call}

A majority of residents approved of 16-hour call duty (65\%). Only a small number preferred 24-hour call (6\%), while the remainder were neutral (28\%) Most residents said that they were doing five or six weekend and overnight calls on average per month, which was slightly higher than for the previous 24-hour call model. There did not appear to be any association between sex, marital status, number of children at home, and year of training in the preference for reduced call models. Despite working more hours per week on average ( $\sim 9$ hours) the results indicated that most residents felt the number of hours they were working was unchanged (60\%), 35\% felt it was reduced and $2 \%$ felt it was increased.

\section{Quality of life}

Most residents indicated that their overall quality of life as either a senior resident (PGY3 or greater) or junior resident (PGY2 and below) had improved (73\% and

Table I Demographic information on McMaster anesthesiology residents participating in the questionnaire $(n=56)$ for the 2013 academic year

Sex

Male $23(58.9 \%)$

Female $33(41 \%)$

$\begin{array}{ll}\text { Residency training year } & 7(12.5 \%) \\ \text { PGYI }\end{array}$

PGY2 $17(30.4 \%)$

PGY3 II (19.6\%)

PGY4 II (19.6\%)

PGY5 $10(17.9 \%)$

Marital status

Single $25(44.6 \%)$

Married/relationship 3I (55.3\%)

Divorced $0(0 \%)$

Children

None $\quad 39(69.6 \%)$

$\mathrm{I}-2 \quad 7(12.5 \%)$

$>2 \quad 10(17.9 \%)$

Self-reported amount of sleep per night

4-6 hours $14(25 \%)$

6-8 hours 4 I (73.2)

$8-10$ hours I (1.8\%)

Abbreviation: PGY, postgraduate years. 
Table 2 Summary of questionnaire results on the effect of 16 hour call

\begin{tabular}{llll}
\hline & Worse & Unchanged & Better \\
\hline Safety of patient care & I (2\%) & $22(49 \%)$ & $22(49 \%)$ \\
Quality of resident education & $10(22 \%)$ & $21(47 \%)$ & $14(31 \%)$ \\
How much rest I get & $0(0 \%)$ & $13(30 \%)$ & $31(70 \%)$ \\
Availability for supervision & $2(4 \%)$ & $37(82 \%)$ & $6(13 \%)$ \\
$\begin{array}{l}\text { Preparation for more senior } \\
\text { residency role }\end{array}$ & $7(15 \%)$ & $31(69 \%)$ & $7(16 \%)$ \\
$\begin{array}{l}\text { Work schedules } \\
\text { Quality of life for senior residents }\end{array}$ & $8(18 \%)$ & $13(29 \%)$ & $24(53 \%)$ \\
Quality of life for interns & $1(3 \%)$ & $11(27 \%)$ & $30(73 \%)$ \\
\hline
\end{tabular}

$55 \%$, respectively). Only one junior resident (3\%) indicated that his/her quality of life was worse. Most residents spent either their pre-call day sleeping (40\%) or studying (45\%), while a minority of residents used that time to spend with family (15\%). Most residents (70\%) spent the majority of time post-call sleeping. If not sleeping, the remainder spent their post-call day with family (11\%), on other activities (11\%), or studying/preparing (9\%) (Figure 1).

\section{Education}

Most residents felt that after the implementation of the 16-hour call schedule they were losing out on good elective cases because of time away from the OR in the daytime prior to their call shift. However, they felt that they were overall seeing more cases because of increased call requirements. During these cases, residents reported feeling more awake, and were able to function and perform better. With respect to one-on-one teaching in the OR and on non-clinical days, most residents ( $47 \%$ ), felt that the quality of resident education was unchanged, $31 \%$ thought it was better, and $22 \%$ felt that it was now worse. Most residents thought that since the implementation of 16-hour call, their preparedness for the transition to a senior resident was unchanged (69\%) (Table 2 ). With respect to being prepared for the board examinations, the results were inconclusive. Some residents thought

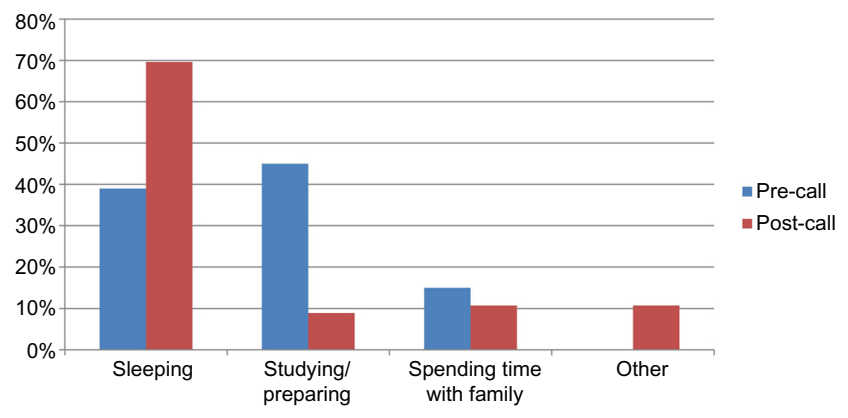

Figure I Comparison of how anesthesia residents spend their time pre- and postcall. that the new call model would help with preparation for the royal college exam (52\%), but others said it would not affect their preparedness at all (47\%).

\section{Patient safety}

Most respondents felt that patient safety had either improved or was unchanged (both 49\%) since the implementation of 16 -hour call. Only $2 \%$ of residents thought that patient safety was worse - however, no other comments were given (Figure 2). That being said, a large number of residents felt that the frequency of patient handovers had increased (44\%), or was unchanged (52\%), compared to 24 -hour call.

\section{Fatigue}

Most residents felt better rested since the implementation of 16-hour call. They indicated that their sleep-wake cycle was less disturbed (despite the fact that they were doing more overnight call duty), and that they now had the ability to rest prior to a shift. Residents acknowledged in the survey that, although not all their time was spent sleeping pre-call, they had time to remove themselves from the stressful environment of the OR and prepare for cases adequately and appropriately. Resident responses also indicated that at 4 am they felt better able to focus.

\section{Discussion}

Our primary finding is that the results support our hypotheses: residents in the department of anesthesia strongly preferred the reduced 16-hour call model over the traditional 24-hour model, and scores on resident wellness, fatigue, education, and patient safety all improved.

The strengths of our study are that it is the first in Canada to present anesthesia residents' perceptions at multiple time periods throughout the year immediately following implementation of a 16-hour call model.

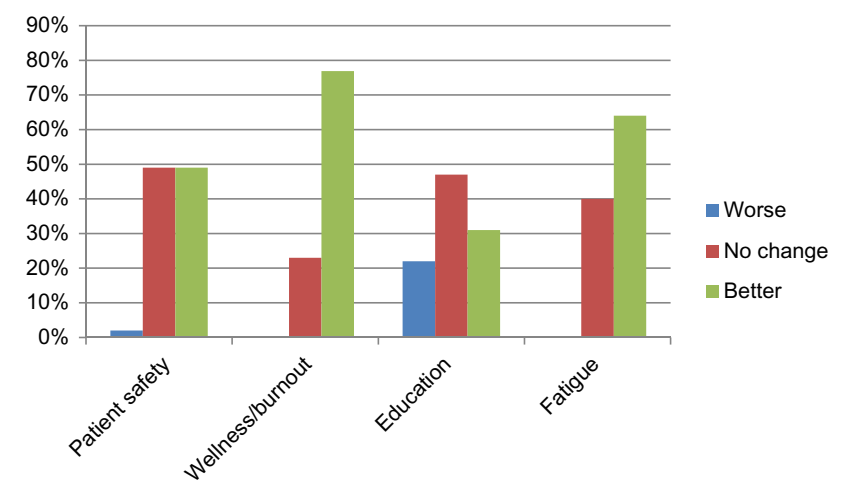

Figure 2 Anesthesia resident opinions on how 16-hour call has affected different factors after the implementation of 16 hour call. 


\section{Resident wellness}

Resident wellness and overall quality of life were perceived as improved with the transition to 16-hour call. A majority of residents preferred the reduced call model to the more traditional 24-hour call model. Even the minority of residents who preferred 24-hour call agreed that since the introduction of 16-hour call their quality of life had also improved. This concurs with the literature. ${ }^{11-13}$

\section{Education}

A majority of residents felt that their educational opportunities had improved, and they were better able to prepare for, and focus on, cases, and the success rates on the board examinations were higher than in previous years. In contrast, in other studies, residents expressed concerns that bedside teaching and opportunities for mentoring by the faculty had decreased after the reform of duty hours, ${ }^{14}$ and duty hour restrictions resulted in a slight decrease in both the quality of teaching and the overall satisfaction with education. ${ }^{15}$ Another study found education after duty hour restrictions to be worse, and attributed it to fewer cases seen and fewer operations being done by surgical residents. Moreover, it pointed to a trend of increased failure rates on the oral component of the surgical board examinations. ${ }^{11}$

The contrast between the literature and our results is probably due to several facts. First, residents are making up for missing more elective OR cases by having more on-call shifts. In the traditional 24-hour call model, residents were doing, on average, 4-5 calls per month; in the new reduced call hour model, residents do 6-7 per month. This has directly increased the number of hours worked on call per month ( 9), which directly translates into more cases seen. Second, the thoracic surgery volume at our center has recently doubled, allowing residents to gain more clinical exposure to thoracic cases. Third, educational opportunities have increased. Since there are fewer learners around during the day, because of pre/ post call days, the availability of rooms with better learning value increases. Junior residents are now getting exposure to more cardiac and thoracic rooms and honing their procedural skills on senior procedures, like thoracic epidurals and Swan-Ganz catheter placement. This is likely the reason why junior anesthesia residents felt more prepared for senior roles, which directly contradicts Drolet et al. ${ }^{10}$

\section{Patient safety and fatigue}

Residents felt that patient safety had improved in the OR in the reduced call model. This is likely related to decreased fatigue and increased vigilance and arousal, resulting in less medical error, and better preparation. No drug errors or near misses were reported. We can infer that giving residents an opportunity to rest prior to starting an on-call shift ought to improve patient safety. The reports on patient safety in the literature have shown that fewer errors result from fatigue, but that more errors are attributable to the loss of continuity of care and the more frequent handovers. ${ }^{15}$ While there is no doubt that 16 -hour call will increase the amount of patient handover in most specialties, this is likely a non-issue in anesthesia. Handover in anesthesia is rare, as it is rare for cases to be interrupted or taken over by a consultant who did not start the case.

\section{Limitations}

Our results may not be fully generalizable to other specialties, since our survey was conducted at one institution with a small number of subjects, and in only one medical specialty $(n=56)$. However, it is the only study to specifically address resident life and patient safety in the field of anesthesia.

The response rate for our survey was $67 \%$. In total there were 84 possible responses ( 28 possible responses at each of the three time intervals). The potential reasons for the reduced response rate were mainly because of vacation and resident post call days. We have no indication that any subset of respondents was unable, or chose not to answer the questionnaire for a reason that would bias our results. Conducting the survey three times avoided entirely missing responses from respondents being too busy at one or another time of the year; the response rate from the PGY5 residents went up dramatically after their board examinations.

The majority of responses in our study were subjective, not objective. However, they are useful for pinpointing current problems, their potential causes, and for developing questions for future quantitative research.

Finally, surveys do not provide definite evidence for the effect of work hour regulations on anesthesia training. There is currently no agreed upon, standardized tool for measuring the impact of these regulations on anesthesia education, and surveys such as ours may be the best metric currently available. In the future, directly comparing subjective measures with objective scores could be beneficial, such as using psychomotor vigilance testing to quantify resident fatigue.

\section{Conclusion}

Our findings strongly suggest that the 16-hour call was preferred by the subjects, and was perceived to improve resident wellness, reduce burnout, and foster an environment where residents were less fatigued and more satisfied with their educational experience, promoting an environment of 
patient safety. The study has several implications: namely, it can inform the active policy debate, guide ongoing implementation of the current duty hour requirements, and direct future policy. We intend to follow-up this cohort throughout the next few years of their training to better understand how duty hour changes further impact the variables: resident wellness and fatigue, patient safety, and resident training and education.

Limiting duty hours represents a necessary paradigm shift in the medical environment, and continued change will take time. As the pendulum on duty hours swings, it is important that we move with it, continuing to teach clinical medicine, but fostering an environment where residents thrive, and patients are safe.

\section{Disclosure}

Funding/Support: the Department of Anesthesia, McMaster University, Hamilton, Ontario. The authors have no other conflicts of interest to report.

\section{References}

1. Sinha A, Singh A, Tewari A. The fatigued anesthesiologist: A threat to patient safety? J Anaesthesiol Clin Pharmacol. 2013;29(2):151-159.

2. Howard SK, Rosekind MR, Katz JD, Berry AJ. Fatigue in anesthesia: implications and strategies for patient and provider safety. Anesthesiology. 2002;97(5):1281-1294.

3. Dawson D, Reid K. Fatigue, alcohol and performance impairment. Nature. 1997;388(6639):235.
4. Gander PH, Merry A, Millar MM, Weller J. Hours of work and fatiguerelated error: a survey of New Zealand anaesthetists. Anaesth Intensive Care. 2000;28(2):178-183.

5. Webb RK, Currie M, Morgan CA, et al. The Australian Incident Monitoring Study: an analysis of 2000 incident reports. Anaesth and Intensive Care. 1993;21(5):520-528.

6. Williamson JA, Webb RK, Sellen A, Runciman WB, Van der Walt JH. The Australian Incident Monitoring Study. Human failure: an analysis of 2000 incident reports. Anaesth Intensive Care. 1993;21(5):678-683.

7. Monk TH. Shiftworker performance. Occup Med. 1990;5(2): 183-198.

8. Denisco RA, Drummond JN, Gravenstein JS. The effect of fatigue on the performance of a simulated anesthetic monitoring task. JClin Monit. 1987;3(1):22-24.

9. Lockley SW, Landrigan CP, Barger LK, Czeisler CA; Harvard Work Hours Health and Safety Group. When policy meets physiology: the challenge of reducing resident work hours. Clin Orthop Relat Res. 2006;449:116-127.

10. Drolet BC, Spalluto LB, Fischer SA. Residents' perspectives on ACGME regulation of supervision and duty hours - a national survey. N Eng J Med. 2010;363(23):e34.

11. Ahmed N, Devitt KS, Keshet I, et al. A systematic review of the effects of resident duty hour restrictions in surgery: impact on resident wellness, training, and patient outcomes. Ann Surg. 2014;259(6):1041-1053.

12. Jamal MH, Rousseau MC, Hanna WC, Doi SA, Meterissian S, Snell L. Effect of the ACGME duty hours restrictions on surgical residents and faculty: a systematic review. Acad Med. 2011;86(1):34-42.

13. Antiel RM, Reed DA, Van Arendonk KJ, et al. Effects of duty hour restrictions on core competencies, education, quality of life, and burnout among general surgery interns. JAMA Surg. 2013;148(5):448-455.

14. Myers JS, Bellini LM, Morris JB, et al. Internal medicine and general surgery residents' attitudes about the ACGME duty hours regulations: a multicenter study. Acad Med. 2006;81(12):1052-1058.

15. Jagsi R, Shapiro J, Weissman JS, Dorer DJ, Weinstein DF. The educational impact of ACGME limits on resident and fellow duty hours: a pre-post survey study. Acad Med. 2006;81(12):1059-1068.
Advances in Medical Education and Practice

\section{Publish your work in this journal}

Advances in Medical Education and Practice is an international, peerreviewed, open access journal that aims to present and publish research on Medical Education covering medical, dental, nursing and allied health care professional education. The journal covers undergraduate education, postgraduate training and continuing medical education

\section{Dovepress}

including emerging trends and innovative models linking education, research, and health care services. The manuscript management system is completely online and includes a very quick and fair peer-review system. Visit http://www.dovepress.com/testimonials.php to read real quotes from published authors. 